Journal of Engineering and Applied Sciences 14 (Special Issue 3): 6150-6155, 2019

ISSN: 1816-949X

(C) Medwell Journals, 2019

\title{
Pupation Preference of Conopomorpha Cramerella (Snellen) after Treated with Zingiber officinale, Curcuma longa and Alpinia galanga Essential Oils
}

\author{
${ }^{1}$ Saripah Bakar, ${ }^{2}$ M.L.S. Noor Hajjar, ${ }^{1}$ A. Alias and ${ }^{3}$ A. Zhang \\ ${ }^{1}$ Malaysian Cocoa Board, 5-7th Floor, Wisma SEDCO, Lorong Plaza Wawasan, Locked Bag 211, \\ 88999 Kota Kinabalu, Sabah, Malaysia, sari@koko.gov.my \\ ${ }^{2}$ Crop Protection Research Group, Faculty of Plantation and Agrotechnology, \\ Universiti Teknologi MARA, 40450 Shah Alam, Selangor, Malaysia \\ ${ }^{3}$ Invasive Insect Biocontrol and Behavior Laboratory, 10300 Baltimore Avenue, BARC-West, \\ Building 007, Beltsville, MD 20705-2350, USA
}

\begin{abstract}
The emergence of Cocoa Pod Borer (CPB), Conopomorpha cramerella Snellen (Lepidoptera: Gracillariidae) in Malaysia was first detected in Tawau, Sabah in 1980. Since then, this pest has become a major threat to cocoa growing countries in the Southeast Asia Region, particularly in Malaysia, Indonesia and Papua New Guinea. Various control efforts were studied, introduced and implemented in Malaysia ranging from biological, chemical, mechanical, host plant resistance and biotechnology aspects. Most of the control approaches from biological aspects were focused on the cocoa black ants, entomophatogenic fungi and microbial, unfortunately, less focus were given to the plant-derived products. Therefore, the study was undertaken to evaluate site pupation preference of C. cramerella using three Essential Oils (EOs) from the Zingiberaceae order that widely planted in Malaysia. The EOs of Zingiber officinale (ginger), Curcuma longa (turmeric) and Alpinia galanga (galanga) were tested on the cocoa pods and observation was carried out in the laboratory. The results show that cumulative means of C. cramerella pupation on cocoa pods was lower compared to cocoa leaves regardless of different treatments through 10 days of observation. Pupation on the cocoa leaves shows significant difference $(\mathrm{p}<0.05)$ on day-2 $(1.653 \mathrm{a})$, compared to days $6,8,9$ and 10 . The results were contradicted with the observation on pods where the highest number of pupation was recorded at day-1 $(0.508 \mathrm{~b})$ and day-10 (0.488 b). Pupa emergence on the cocoa pod surface and cocoa leaves from day-1 through day-10 shows the highest mean of pupation was observed at control treatment in all daily observations, except for day-2. Pupa emergence on cocoa pods was observed to have significantly different $(\mathrm{p}<0.05)$ at control $(0.324 \mathrm{a})$, compared to Z. officinale $(0.556 \mathrm{~b})$, C. longa $(0.580 \mathrm{~b})$ and $A$. galanga $(0.641 \mathrm{~b})$. No significant different denoted among EOs on the pupation on cocoa pods. Throughout the observation among EOS, Z. officinale recorded the lowest pupation both for cocoa pods $(0.556 \mathrm{~b})$ and cocoa leaves $(0.655 \mathrm{c})$. The results may suggest that the EOs of Zingiberaceae, particularly the $Z$. officinale may have a positive effect and can be a part of botanical pesticide formulation in the future.
\end{abstract}

Key words: Cocoa, theobroma cacao, cocoa pod borer, Conopomorpha cramerella, Zingiberaceae, pesticide

\section{INTRODUCTION}

Cocoa, Theobroma cacao (Linnaeus) (Malvales: Sterculiaceae) is a crop that widely distributed throughout Malaysia, Indonesia, the Philippines and Papua New Guinea. The omnipresence of Cocoa Pod Borer (CPB), Conopomorpha cramerella (Lepidoptera: Gracillariidae), emerged as the most important pest of cocoa wherever it planted. The multivoltine insect has caused declines in the cocoa industry in Malaysia. The larval stage of holometabolous C. cramerella feed on mucilage and placenta for their development. Entire larval stages are inside the pod and almost fully protected from any control approach. Infested cocoa beans were hardened clumped together that leads to difficulty in extracting beans. Mature larva emerged fromcocoa pod for pupation, immediately constructing the silken cocoon that eclosed their entire body part. The cocoon was usually found intact on the dry cocoa leaves, green leaves or on pod surface. The economic losses to farmers can be substantial and continuous infestations may cause an unacceptable level of damage (Saripah, 2014).

Corresponding Author: Saripah Bakar, Malaysian Cocoa Board, 5-7th Floor, Wisma SEDCO, Lorong Plaza Wawasan, Locked Bag 211, 88999 Kota Kinabalu, Sabah, Malaysia, sari@koko.gov.my 


\section{J. Eng. Applied Sci., 14 (Special Issue 3): 6150-6155, 2019}

Since, C. cramerella first discovered in Malaysia, diverse attempts have been undertaken to manage the infestation which includes rampasan, pod sleeving, frequent harvesting of ripe pods, utilization of natural enemies, quarantine measures and insecticide spraying (Ooi et al., 1987). Among these, biweekly prophylactic treatment with chemical insecticides was considered as one of the most effective approaches (Alias, 2011). However, the effectiveness of insecticide largely depends on the appropriate timing of spraying (Azhar et al., 2000). Necessitates management in order to suppress the pest population, like performing an additional number of insecticide sprays may lead to increase of residue level and input costs. Prolonged use of chemicals may risk of insecticide resistance due to the excessive use. Insects, after treating with insecticides or other treatment may pose abnormal phenotypes or multiple symptoms at their different life stages, if the applied doses were unable to causing quick mortality (Huang et al., 2015). Development of resistance towards insecticide can be best referred to the Lepidopteran pest of agriculture, Spodoptera litura where many field populations have developed resistance to multiple insecticides (Liu et al., 2018). Sublethal developmental effects of treatment to the Lepidopteran may include larval body weight, decreased pupation and emergence as well as notched wings in adults (Liu et al., 2018). Individual larva of $S$. litura that treated with fluralaner insecticide shows symptoms like smaller body weight, decreased in the activity as well as reduce appetite. The survival pupa may show multiple abnormal phenotypes like smaller in size, change in coloration as well as sunken puparium. Meanwhile adults that developed from treated life stages may generate abnormal adult, pupa-adult intermediate, displayed a notched or deform phenotype or failed to emerge.

Preference for site pupation is also important where suitable microhabitat is significant in influencing pupation behaviors and pupal survivorship (Wang et al., 2018). Selection of pupation site is crucial for holometabolous insect, in order to ensure successful emergence of the adults. As in for the Drosophila melanogaster, the interaction between biotic and abiotic factors will influence on pupation behavior and will inflict the fitness on an insect later on (Beltrami et al., 2010). The distance between the substrate surface and the pupation site will be an indicator for survival strategy and selection of the site preference. For temperate insect-like pine sawflies (Diprion similis) selection of inaccessible position during the Summer or fall which less likely to be attacked will reduce the probability of predation during the Winter and later on increase their survival rate (Wheelwright et al., 2017). Information on the pupation site is also beneficial for the selection of any cultural or management programs for pest control (Woltz and Lee, 2017). Numerous studies on the pupation subjects, i.e., activities, behavior, effects of insecticides, laboratory and under nature pupation, site selection, food preference, natural enemies and other interesting aspects had been carried out on multiple insects, especially the economical pests (Bakar et al., 2017; Beltrami et al., 2010; Delgado-Tejeda et al., 2016; Liu et al., 2018; Qu et al., 2015; Shintani et al., 2017; Wang et al., 2018; Woltz and Lee, 2017).

As an alternative to chemical pesticides, an effort is warranted to find a new environmentally friendly approach to currently used insecticides. One of the approaches that should be taken into consideration is the use of natural products synthesized by plant species. In controlling Drosophila suzikii, for example, there are already few effective, organic insecticides in the market (Woltz and Lee, 2017). Many plant products have been identified to have potential effects as oviposition deterrent, insect repellent or antifeedant via. larvicidal, pupicidal or adulticidal form. To date more than 800 plant species pose insecticidal, repellent and anti-feeding effects were used in agriculture and this will offer more variety of selections in the future (Parkash and Rao, 1997). With more than 1,000 species recorded, Zingiberaceae (Order: Zingiberales) is one of the largest families in the plant kingdom. Further, Zingiberaceae is recognized as the most important herbaceous species in the tropics with approximately 50 genera (Sirirungsa, 1998). Zingiberaceae species which are domesticated in the Southeast Asia Region have potential effects as a botanical pesticide towards several orders of insect pests. Unfortunately, apart from the great distribution in Malaysia, little is known about the use of the Zingiberaceae as a botanical pesticide in this country. Moreover, less is known about the potential of Zingiberaceae plants for controlling the insect pest of cocoa, particularly C. cramerella. As part of a current research which aims at studying the potential of Zingiberaceae in managing C. cramerella, the goal of this study is to evaluate the effect on pupation preference of C. cramerella after treating with a sublethal dose of Essential Oils (EOs) from three Zingiberaceae species; Zingiber officinale (ginger), Curcuma longa (turmeric) and Alpinia galanga (lesser galanga).

\section{MATERIALS AND METHODS}

Pupation behavior of C. cramerella was evaluated when treated with Zingiberaceae EOs under the laboratory condition of the Malaysian Cocoa Board, Cocoa Research and Development Center (CRDC), Bagan Datuk, Perak $(3.906 \mathrm{~N}, 100.866 \mathrm{E})$. Zingiberaceae EOs were purchased 
from the local authorized dealer and diluted with water mixed with Tween 80 at the concentration of $400 \mathrm{ppm}$. A similar amount of water and Tween 80 were used as control. Concentrations were vortex (2 $\mathrm{min}$ at $1800 \mathrm{rpm})$, stored in an amber dark glass bottle at $4^{\circ} \mathrm{C}$ for $24 \mathrm{~h}$ prior to the experiment.

Mature cocoa pods from untreated cocoa field approximately 4-5 months old with prominent symptoms of C. cramerella were selected for this study. The selected pods were divided into four different treatments. Forty pods for each treatment were hung, sprayed, accordingly to the treatment using a hand sprayer at a range of $15 \mathrm{~cm}$ and then leave for air-dried for 1-2 h. Further, 10 pods were placed as a single layer in the small plastic container where four containers were prepared for each treatment (10 pods/containerx 4 containersx 4 treatments). The pods were covered with dry cocoa leaves for pupation sites. Pupa that pupates on the pod surface and dry cocoa leaves were recorded on a daily basis until day-10. Bioassays were repeated five times for each treatment. For observation on the length reduction, five newly-emerged pupae were selected randomly from each treatment. Measurement of pupa length was carried out at day-1 and 5 using the Dino-Lite Premier handheld digital microscope at the magnification of 30x (Dino Capture 2.0, Model AM7013MZT). The percentage of reduction was calculated based on pupa length at day-1 and 5 old. Experimental data were arranged separately based on the treatments and replicates in Microsoft $\circledast$ Excel 2007. All data were subjected to statistical analysis and significant differences were analyzed in SAS Software from SAS ${ }^{\circledR}$ Version 8 by a Duncan's Multiple Range Test (DMRT).

\section{RESULTS AND DISCUSSION}

The study noticed that in order wandering for pupation site as well as for an escapism strategy, C. cramerella larva crawls for a few centimeters after emerging from the cocoa pod before pupate either on the pod surface or hide under the leaf. No aggregation of the pupation sites was found on the pods as well as on the leaves. The pupation on the cocoa pod surface (Table 1) was the highest at day-4 for $Z$. officinale $(0.350 \pm 0.48)$, day-6 $(0.300 \pm 1.13)$ for $C$. longa and day- 7 and 8 for A. galanga. Emergence of $C$. cramerella pupae was continued until day-10 where highest mean was recorded on day-8 (2.067 \pm 3.06$)$. Pupation on leaves usually conspecific either on the adaxial surface or side facing the treated pod. Mean of pupation recorded the highest at day-6 for control $(2.933 \pm 2.96)$ and day-2 for all EOS; Z. officinale $(1.050 \pm 1.14)$, C. longa (2.500 \pm 2.98$)$ and A. galanga (1.300 \pm 1.49$)$.

Assays evaluating site pupation preference shows that the CPB pupa prefers to pupate on cocoa leaves as compared to pod for all treatments (Fig. 1). Pupation on the cocoa leaves exert significant effect $(\mathrm{p}<0.05)$ on day-2 (1.653 a), compared to day-6 (1.093 bed), day-8 $(0.973 \mathrm{bcd})$ and day- $9(0.946 \mathrm{~cd})$. Low pupation was occurred on day-10 (0.626 d). The results was contradicted with the pupation on cocoa pods where highest pupation was recorded at day-1 $(0.508 \mathrm{~b})$ and day-10 (0.488 b). High pupation on cocoa leaves suggest that most of the mature larva emerged from cocoa pod will find a suitable place for pupation. The reason why the dry cocoa leaves more preferred by $C$. cramerella might be due treatment was not directly spray onto the surface. Therefore, no or only little less treatment residue on the leaf. Less of moisture might also a factor contributing to this preference where cocoa pod surface harbored more moisture compared to the dry cocoa leaf. Excessive moisture in a substrate or surface may become abiotic factor that influence low emergence of Lepidoperan Comadia redtenbacheri in the nursery (Delgado-Tejeda et al., 2016). Likewise, extremely low or high medium such as the soil moisture, significantly decreased the emergence of various soil-pupating insects (Wang et al., 2018). C. cramerella might also tend to pupate on the safer surface, like pupation on the outside part or the plastic containers as recorded several times in this study. It was in agreement with the observation by Wang et al. (2018) where Lepidopteran

Table 1: The pupation of C. cramerella on the cocoa pod surface

\begin{tabular}{|c|c|c|c|c|c|c|c|c|c|}
\hline \multirow[b]{2}{*}{$\begin{array}{l}\text { Day after } \\
\text { treated }\end{array}$} & \multirow[b]{2}{*}{$\mathrm{n}$} & \multicolumn{4}{|c|}{ Pupation on cocoa pod } & \multicolumn{4}{|c|}{ Pupation on cocoa leaves } \\
\hline & & Z. officinale & C. longa & A. galonga & Control & Z. officinale & C. longa & A. galonga & Control \\
\hline Day-1 & 20 & $0.00 \pm 0.00$ & $0.000 \pm 0.00$ & $0.050 \pm 0.22$ & $1.000 \pm 1.00$ & $0.900 \pm 0.78$ & $1.028 \pm 1.94$ & $1.600 \pm 1.35$ & $1.600 \pm 1.35$ \\
\hline Day-2 & 20 & $0.300 \pm 0.65$ & $0.150 \pm 0.67$ & $0.000 \pm 0.00$ & $1.667 \pm 1.34$ & $1.050 \pm 1.14$ & $2.500 \pm 2.98$ & $1.300 \pm 1.49$ & $1.800 \pm 1.32$ \\
\hline Day-3 & 20 & $0.050 \pm 0.22$ & $0.150 \pm 0.49$ & $0.000 \pm 0.00$ & $1.600 \pm 1.24$ & $1.300 \pm 0.73$ & $1.350 \pm 0.81$ & $1.250 \pm 1.16$ & $2.200 \pm 1.69$ \\
\hline Day-4 & 20 & $0.350 \pm 0.48$ & $0.150 \pm 0.37$ & $0.050 \pm 0.22$ & $0.933 \pm 0.89$ & $0.80 \pm 1.05$ & $0.450 \pm 0.81$ & $0.950 \pm 1.35$ & $2.267 \pm 1.43$ \\
\hline Day-5 & 20 & $0.100 \pm 0.31$ & $0.100 \pm 0.45$ & $0.005 \pm 0.22$ & $1.267 \pm 1.39$ & $0.651 \pm 0.74$ & $0.900 \pm 0.71$ & $0.900 \pm 1.29$ & $2.333 \pm 1.58$ \\
\hline Day-6 & 20 & $0.200 \pm 0.41$ & $0.300 \pm 1.13$ & $0.005 \pm 0.22$ & $1.867 \pm 2.17$ & $0.553 \pm 0.68$ & $0.400 \pm 0.88$ & $0.950 \pm 1.05$ & $2.933 \pm 2.96$ \\
\hline Day-7 & 20 & $0.000 \pm 0.00$ & $0.200 \pm 0.41$ & $0.100 \pm 0.45$ & $1.200 \pm 1.42$ & $0.700 \pm 0.05$ & $0.650 \pm 0.67$ & $0.700 \pm 1.03$ & $2.667 \pm 1.79$ \\
\hline Day-8 & 20 & $0.300 \pm 0.47$ & $0.200 \pm 0.70$ & $0.100 \pm 0.45$ & $2.067 \pm 3.06$ & $0.750 \pm 0.91$ & $1.000 \pm 1.12$ & $0.350 \pm 0.58$ & $2.067 \pm 1.79$ \\
\hline Day-9 & 20 & $0.000 \pm 0.00$ & $0.050 \pm 0.22$ & $0.100 \pm 0.45$ & $1.200 \pm 1.21$ & $0.600 \pm 0.68$ & $1.250 \pm 1.55$ & $0.300 \pm 0.65$ & $1.867 \pm 1.95$ \\
\hline Day-10 & 20 & $0.000 \pm 0.00$ & $0.500 \pm 0.22$ & $0.050 \pm 0.22$ & $1.133 \pm 1.30$ & $0.250 \pm 0.44$ & $0.600 \pm 0.75$ & $0.250 \pm 0.44$ & $1.667 \pm 1.63$ \\
\hline
\end{tabular}

Bold values are significant 


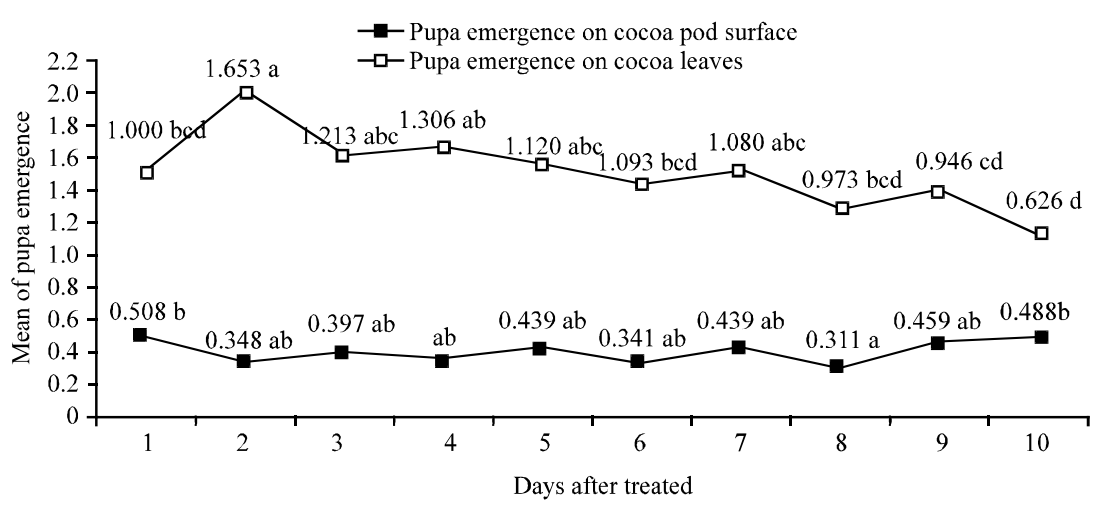

Fig. 1: Pupa emerging on the cocoa pod surface and cocoa leaves ${ }^{a}$ values by the same letters are not significant $(\mathrm{p}>0.05)$ different in Duncan's multiple range test

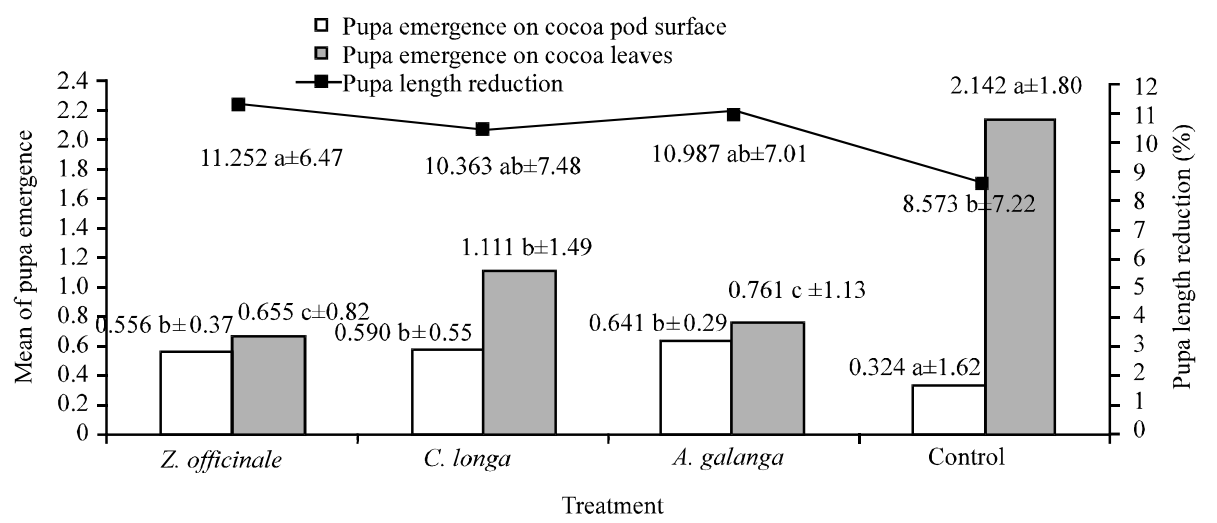

Fig. 2: The pupation on cocoa pod and leaves for different treatments and the pupa length reduction. ${ }^{a}$ Each value is expressed as mean $\pm \mathrm{SD}$. ${ }^{\mathrm{B}}$ values by the same letters of the same color are not significant $(\mathrm{p}>0.05)$ different in Duncan's multiple range test

Ectropis grisescens able to pupate in a plastic container, even when there was lack of pupation substrate was insufficient.

Pupa emergence on the cocoa pod surface and cocoa leaves from day-1 through day-10 shows the highest mean of pupation was observed in the control treatment (Fig. 2). The result ( $2.142 \mathrm{a} \pm 1.80$ ) was significantly higher $(\mathrm{p}<0.05)$ than C. longa $(1.111 \mathrm{~b} \pm 1.49)$, A. galanga $(0.761$ $\mathrm{c} \pm 1.13)$ and $Z$. officinale $(0.655 \mathrm{c} \pm 0.82)$. Pupation on cocoa pods for all Zingiberaceae EOs were significantly different compared with control $(0.324 \mathrm{a} \pm 1.62)$. Nevertheless, the number of pupation on the pods did not exert a significant effect among the Zingiberaceae EOs. Meanwhile assays evaluating of pupa length revealed that control recorded the lowest percentage of length reduction $(8.573 \mathrm{~b} \pm 7.22)$ and shows the different compared to $Z$. officinale (11.252 a \pm 6.47 ).

Multiple sublethal effects may resulted after pests were exposed to the sublethal dose circumstances and can be observed while testing new insecticide or treatment (Qu et al., 2015). The sublethal effects may include the feeding behavior, developmental rate, immune capacity and many other aspects. Currently, very less or probably no study focused on the treatment effects to the site pupation of $C$. cramerella. Presence of suitable substrate is critical to complete the pupal-adult metamorphosis (Wang et al., 2018). Compared to the control, Z. officinale, C. longa and A. galanga were able to influenced site preference of $C$. cramerella to pupate. During the bioassays, $Z$. officinale recorded the lowest pupation both for cocoa pods $(0.556 \mathrm{~b} \pm 0.37)$ and cocoa leaves $(0.655 \mathrm{c} \pm 0.82)$ among other two EOs, even at the same concentration $(400 \mathrm{ppm})$. The results may indicate that $Z$. officinale influences the pupation sites where the pre-pupa tends to wander away from the treated pods, and subsequently pupate on the dry cocoa leaves. The presence of Zingiberaceae EOs residue on the treated pods may reduced pupation on pods. Similar effect of $Z$. officinale, C. longa and A. galanga were also observed on the $C$. cramerella pupa as previously discussed by 
Bakar et al. (2017). Based on the reduction of body length and adult deformities, they denote that $Z$. officinale shows the most promising results among the EOS for observation on both parameters.

The effectiveness of Zingiberaceae EOs, particularly $Z$. officinale on C. cramerella pupa can be seen with the reduction in pupa length as compared to control. In our study, we did not observe the pupal weight, due to the entire body was enclosed inside the cocoon. Removing the cocoon will expose the pupa to the environment and for the newly emerged pupa, they usually able to re-construct a new cocoon as soon as the cocoon was disrupted. In contrast with older pupa, they usually unable to move, become dry and die several hours after removal of the cocoon. To reduce the risk, observation on the pupa was carried out using the length and it also shows a negative effect after treated with the treatment. The length becomes shorter and the reduction of size was greater for treated pupae. This result supports the observation made by Liu et al. (2018) when S. litura larvae was treated with fluralaner insecticide where individual larvae displayed some typical symptoms, including reduced appetite, decreased activity and smaller body weight. On the other hand, the larva may also tend to pupate precociously with a smaller body size if they are deprived of food (Shintani et al., 2017). But in this study, the effect of a food source can be discarded, since, the cryptic habit of $C$. cramerella where they spend their entire larval stage inside the cocoa pod, thus, lack of food may not be the factor in reducing the pupa length. The symptoms almost similar with the observation on the thick mean weight of Ixodes ricinus and Ixodes scapularis. Both ticks weight were lighter compared with control when treated with insecticide. Zingiberaceae EOs may also able to suppress the proportion of individual pupae to the adults but the results were not reported in this study.

\section{CONCLUSION}

An effort has been made to find a new alternative to insecticides for controlling C. cramerella damage on cocoa. This includes the use of natural products synthesized by plant species. The effects of plant extracts, Zingiberaceae EOs on pupation site of C. cramerella pupa has been demonstrated in this study. Mature larva emerged from cocoa pod preferred to pupate on dry leaves rather than cocoa pod surface after the pod were treated with Zingiberaceae EOs at a concentration of $400 \mathrm{ppm}$. Similarly, pupa collected from Zingiberaceae EOs is slightly shorter than the pupa from cocoa pod and subsequently influence the size of adult moths and its population behavior. This finding warrants further study on the potential use of Zingiberaceae Eos in managing C. cramerella, the most devastating pest of cocoa in the Southeast Asia Region.

\section{ACKNOWLEDGEMENTS}

The researcher sincerely thank the Director General of Malaysian Cocoa Board, Datin Norhaini Udin for the permission to conduct this study. We also thank technical assistance by the Entomology staffs at CRDC Bagan Datuk and Jengka; Mr. Sulaiman Hashim, Mr. Roslan Sa'adi, Mr. Ahmad Zaki Yusoff, Mr. Abdul Mutalib Abd. Kadir, Mr. Mohamad Faiz Yahya and Mr. Abdul Rahman Mohd Isa. The study on the Zingiberaceae is fully supported by the Temporary Research Fund, Malaysian Cocoa Board (L15288).

\section{REFERENCES}

Alias, A., 2011. Resistance mechanisms in cocoa to cocoa pod borer. Ph.D Thesis, University of Reading Reading, UK.

Azhar, I., A. Alias and M.Y. Meriam, 2000. Research on the cocoa pod borer in Malaysia. Proceedings of INCOPED 3rd International Seminar, October 16-17, 2000, Malaysian Cocoa Board, Kota Kinabalu, Sabah, Malaysia, pp: 105-113.

Bakar, S., L. Snhm, A. Awang and A. Zhang, 2017. Effects of Zingiber officinale, Curcuma longa and Alpinia galangal essential oils on the morphological characteristic of cocoa pod borer, Conopomorpha cramerella. J. Fundam. Appl. Sci., 9: 25-38.

Beltrami, M., M.C. Medina-Munoz, D. Arce and R. Godoy-Herrera, 2010. Drosophila pupation behavior in the wild. Evol. Ecol., 24: 347-358.

Delgado-Tejeda, I., C. Llanderal-Cazares, K. Miranda-Perkins and H.M.D.L. Santos-Posadas, 2016. Pupation, adult emergence and oviposition of Comadia redtenbacheri (Lepidoptera: Cossidae) in the nursery. Agrociencia, 51: 447-454.

Huang, Z., Y. Wang and Y. Zhang, 2015. Lethal and sublethal effects of cantharidin on development and reproduction of Plutella xylostella (Lepidoptera: Plutellidae). J. Econ. Entomol., 108: 1054-1064.

Liu, D., Z.Q. Jia, Y.C. Peng, C.W. Sheng and T. Tang et al., 2018. Toxicity and sublethal effects of fluralaner on Spodoptera litura Fabricius (Lepidoptera: Noctuidae). Pestic. Biochem. Physiol., 152: 8-16.

Ooi, P.A.C., G.C. Luz, K.C. Khoo, C.H. Teoh and M. Jusoh et al., 1987. Management of the Cocoa Pod Borer. Malaysian Plant Protection Society, Kuala Lumpur, Malaysia, ISBN:9789679942088, Pages: 192. 
Prakash, A. and J. Rao, 1997. Botanical Pesticides in Agriculture. CRC Press Inc., USA., ISBN-13: 9780873718257, Pages: 480.

Qu, Y., D. Xiao, J. Li, Z. Chen and A. Biondi et al., 2015. Sublethal and hormesis effects of imidacloprid on the soybean aphid Aphis glycines. Ecotoxicol., 24: 479-487.

Saripah, B., 2014. Control of cocoa pod borer using insecticides and cocoa cacao black ants. Malaysian Cocoa J., 8: 14-22.

Shintani, Y., M. Terao and S. Tanaka, 2017. Adaptive significance of precocious pupation in the bean blister beetle, Epicauta gorhami (Coleoptera: Meloidae), a hypermetamorphic insect. J. Insect Physiol., 99: 107-112.
Sirirugsa, P., 1998. Thai Zingiberaceae: Species diversity and their uses. Pure Appl. Chem., 70: 98-110.

Wang, H., S. Liang, T. Ma, Q. Xiao and P. Cao et al., 2018. No-substrate and low-moisture conditions during pupating adversely affect Ectropis grisescens (Lepidoptera: Geometridae) adults. J. Asia Pac. Entomol., 21: 657-662.

Wheelwright, N.T., L.U. Taylor, B.M. West, E.R. Voss and S.Y. Berzins et al., 2017. Pupation site selection and enemy avoidance in the introduced pine sawfly (Diprion similis). Northeastern Nat, 24: 19-31.

Woltz, J.M. and J.C. Lee, 2017. Pupation behavior and larval and pupal biocontrol of Drosophila suzukii in the field. Biol. Control, 110: 62-69. 